\title{
Utilidad de la TAC secuencial y la monitorización de la presión intracraneal para detectar nuevo efecto masa intracraneal en pacientes con traumatismo craneal
} grave y lesión inicial Tipo I-II

\author{
R.D. Lobato; J.F. Alen; A. Perez-Nuñez; R. Alday; P.A. Gómez; B. Pascual; A. Lagares; P. Miranda, I. Arrese y A. Kaen
}

Servicio de Neurocirugía. Hospital 12 de Octubre, Madrid.

Resumen:

Objetivo. Analizar los cambios en la patología y presión intracraneal (PIC) durante el periodo agudo postraumático en una serie de pacientes con trauma craneal grave y lesiones Tipos I-II en la TAC inicial (clasificación del Traumatic Coma Data Bank) con el objetivo de diseñar la pauta mas adecuada de uso de TAC secuencial y monitorización de la PIC para detectar nuevo efecto masa intracraneal y tratar así de mejorar la evolución final de pacientes.

Material y métodos. Se analiza una serie de 56 pacientes $($ edades $=15-80$ años) admitidos consecutivamente en un periodo de dos años que fueron sometidos a TAC inicial $<24$ horas tras el impacto, (intervalo medio $=150$ minutos), TACs control en los primeros días del curso, y monitorización de la PIC. Se recogieron diferentes variables epidemiológicas, clínicas, radiológicas y se consideró como variable dependiente el desarrollo de deterioro definido como elevación mantenida de la PIC por encima de $20 \mathrm{mmHg}$ que requiriera tratamiento agresivo médico y/o quirúrgico. Mediante análisis bi y multivariante se determinaron las correlaciones entre las diferentes variables y la aparición de deterioro. Para estimar la afectación neurológica y el resultado final se emplearon las escalas de coma y evolución de Glasgow, respectivamente.

Resultados. El "score " medio en la serie fue de 5, y $37 \%$ de los pacientes tuvieron cambios pupilares, $52,3 \%$ hipotensión-hipoxemia, $16.1 \%$ anemia peritraumáticas y $12,3 \%$ alteraciones de la coagulación. $50 \%$ de los pacientes mostraron petequias en sustancia blanca y/o tronco cerebral en la TAC inicial, $66 \% \mathrm{HSA}, 40 \% \mathrm{HIV}$, $39,3 \%$ contusión y $21,4 \%$ hematomas extraaxiales. $57,1 \%$ de los pacientes mostraron cambios en la TAC de control consistentes en nueva contusión en $26,8 \%$ de los casos, crecimiento de contusión previa en $68,2 \%$, crecimiento de hematoma previo en $10,7 \%$ y swelling cerebral generalizado en $10,7 \%$. $64 \%$ de los pacientes

Recibido: 10-09-04. Aceptado: 11-11-04 experimentaron una evolución final favorable y $35,7 \%$ desfavorable.

27 pacientes $(48,9 \%)$ desarrollaron deterioro PIC, de los que $21(37,5 \%)$ presentaron cambios concurrentes en la TAC, y $6(10,7 \%)$ no los mostraron. Los restantes $29(51,7 \%)$ pacientes no presentaron deterioro PIC, aunque $11(19,6 \%)$ de ellos mostraron cambio TAC. La edad, el "score", la presencia de hipotension-hipoxemia peritraumáticas y los trastornos de la coagulación no se correlacionaron con riesgo de deterioro. Por el contrario, la presencia de contusión inicial $(\mathrm{p}=\mathbf{0 , 0 1})$ y el cambio TAC (en forma de desarrollo de swelling cerebral generalizado, $p=0,003$ ) se correlacionaron con la aparición de deterioro; a su vez el deterioro multiplicó por $10(\mathrm{OR}=9,8)$ el riesgo de muerte y 7 de los 8 pacientes que fallecieron desarrollaron hipertensión intracraneal intratable. Los 8 pacientes $(14,2 \%)$ que necesitaron cirugía evacuadora o descompresiva presentaron simultáneamente cambio PIC y cambio TAC, si bien otros 13 en situación similar pudieron ser manejados sin cirugía. Mostraran o no deterioro PIC, los pacientes sin cambio TAC evolucionaron mejor que los que desarrollaron nuevas patologías, pero la diferencia no alcanzó diferencia significativa.

Discusión y conclusiones. Más de la mitad de los pacientes con lesión inicial Tipo I-II desarrolla cambios patológicos secuenciales, y casi el $\mathbf{5 0 \%}$ presenta hipertensión intracraneal. Dada la alta incidencia de cambios TAC y PIC, la escasez y debilidad de los factores predictores de dichos cambios, y la frecuente discordancia entre ambos tipos de cambio $(30,3 \%$ de los casos), parece recomendable monitorizar la PIC desde el inicio y practicar TACs 2-4, 12, 24, 48 y 72 horas tras el impacto en todos los pacientes, y otros adicionales si

Abreviaturas. ECG: escala de coma de Glasgow. HIC: hipertensión intracraneal. HIV: hemorragia intraventricular. HSA: hemorragia subaracnoidea. PIC: presión intracraneal. TAC: tomografia axial computarizada. TCDB: traumatic coma data bank. TCG: traumatismo craneal grave. TTPA: tiempo parcial de tromboplastina activada. 
la evolución clínica o de la PIC lo requiriera.

Si bien parece indudable que el desarrollo de hipertensión intracraneal grave incrementó significativamente el riesgo de muerte, la escasez de la muestra en la serie no permite determinar la contribución del nuevo efecto masa y/o la elevación de la PIC al desarrollo de incapacidad moderada y grave en los pacientes que no fallecieron, causada principalmente por la lesión axonal difusa. Finalmente, demostrar que la practica de TAC secuencial y la monitorización de la PIC mejoran la evolución final de este tipo de pacientes requeriría un estudio prospectivo aleatorizado que no es practicable por diferentes razones, entre ellas las de tipo ético.

PALABRAS CLAVE: Trauma craneal. Coma. Contusión cerebral. Hematoma epidural. Lesión axonal difusa. Tomografía computarizada. Presión intracraneal.

Value of serial CT scanning and intracranial pressure monitoring for detecting new intracranial mass effect in severe head injury patients showing lesions type I-II in the initial CT scan

\section{Summary}

Objective. To determine the incidence of pathological and intracranial pressure (ICP) changes during the acute posttraumatic period in severe head injury patients presenting with lesions Types I-II (TCDB classification) in the admission CT scan with the aim of defining the most appropriate strategy of sequential CT scanning and ICP monitoring for detecting new intracranial mass effect and improving the final outcome.

Material and methods. 56 patients (ages 15-80 years) consecutively admitted during a 2 years period were included. All had the initial CT scan $<24$ hours after injury (mean interval $=150 \mathrm{~min}$ ), several $\mathrm{CT}$ controls within the first days of the course and ICP monitoring after admission. Different epidemiological, clinical and radiological variables were recorded and deterioration defined as the development of sustained ICP over 20 mmHg requiring aggressive medical and/or surgical treatment was considered the dependent variable. Uni and multivariate analyses were made for determining the correlation between different parameters and the occurrence of deterioration and the final outcome as assessed with the GOS.

Results. The mean GCS score was 5 and $37 \%$ of the patients showed pupillary changes; $52.3 \%$ had peritraumatic hypotension-hypoxemia, $16.1 \%$ anemia and $12.3 \%$ coagulation changes. $50 \%$ of the patients showed petechial hemorrhages in the white matter or the brainstem, 66\% SAH, 40\% HIV, 39.3\% brain con- tusion and $21.4 \%$ small extraxial hematomas. $57.1 \%$ of the patients showed CT changes through the acute posttraumatic period consisting of new contusion $(26.8 \%$ of the cases), growing of previous contusion (68.2\%) or previous extraaxial hematoma $(\mathbf{1 0 . 7 \%})$, and generalized brain swelling $(\mathbf{1 0 . 7 \%}) .64 .9 \%$ of the patients made a favourable and $35.7 \%$ an unfavourable outcome.

Overall, $27(48.9 \%)$ patients developed deterioration, $21(37.5 \%)$ with concurrent CT changes and 6 $(\mathbf{1 0 , 7 \% )}$ without new pathology as seen by the CT control. The remaining $29(51.7 \%)$ patients in this series did not develop deterioration in spite that 11(19.6\%) showed CT changes. The age, the initial score, the occurrence of peritraumatic hypotension-hypoxemia and coagulation disorders did not correlate with the risk of deterioration. By contrast, the presence of contusion at the initial CT scan $(p=0.01)$ and the occurrence of CT change (only generalized brain swelling, $p=0.003$ ) significantly correlated with the risk of deterioration; in his turn deterioration increased by a factor of $10(\mathrm{OR}=9,8)$ the risk of death and 7 out of the 8 patients who died developed intractable intracranial hypertension. The $8(14.2 \%)$ patients requiring surgery showed simultaneous ICP deterioration and CT changes, but another 11 patients in a similar condition could be managed without surgery. With or without ICP deterioration, patients showing CT changes had a worse outcome than those without new pathologies, but the difference did not reach statistical significance,

Discussion and conclusions. Over $50 \%$ of the patients with initial Type I-II lesions developed new CT changes and nearly $50 \%$ showed intracranial hypertension during the acute posttraumatic period. Considering the high incidences of ICP and CT deterioration through the course, along with the absence of strong predictors and the discordances between CT and ICP changes (which were seen in $30.3 \%$ of the cases) we recommend ICP monitoring after admission in all patients and serial CT scanning at 2-4, 12, 24, 48 and 72 hours after injury with additional controls as indicated by clinical or ICP changes in all cases.

Though it is clear that the presence of severe intracranial hypertension significantly increased the risk of death, the small size of the sample in this series prevented to assess to what extent the occurrence of new mass effect and/or raised ICP contributed to the development of moderate and severe disability in the survivors which were mainly due to the occurrence of diffuse axonal injury. Finally, demonstrating that sequential CT scanning and ICP monitoring improve the final outcome in this type of patients would require a prospective randomized trial which is impracticable for different reasons, among them the ethical ones. 
KEY WORDS: head injury. Postraumatic coma. Brain contusion epidural hematoma. Axonal injury. Computerized tomography. Intracranial pressure.

\section{Introducción}

Las lesiones difusas Tipos I-II definidas según la clasificación patológica del Traumatic Coma Data Bank $(\mathrm{TCDB})^{19}$, representan alrededor del $7 \%$ y el $24 \%$, respectivamente del total de los casos de traumatismo craneal grave (TCG) en diferentes series ${ }^{6,15,19}$ y conllevan un riesgo de mortalidad que oscila entre el $5 \%$ y el $16 \%$ en los pacientes con lesión inicial tipo I, y alcanza el $21-27 \%$ en aquellos con lesiones tipo II, tras excluir un reducido número de pacientes que fallecen por causas extraneurológicas ${ }^{15,19}$.

Durante la era angiográfica y los primeros años de la era TAC se pensó que los pacientes con TCG que no mostraban efecto masa focal en el examen inicial tenían un riesgo mínimo de desarrollar elevación de la presión intracraneal (PIC), y que su evolución estaba condicionada casi exclusivamente por la intensidad de la lesión axonal difusa sufrida tras el impacto. Sin embargo, pronto se comprobó que aparte de la influencia usualmente determinante de la lesión axonal, la evolución final de estos pacientes podía empeorar a causa de la aparición demorada de nuevas lesiones intracraneales susceptibles de diagnóstico y tratamiento, lo que indicaba la importancia de disponer de la mejor estrategia diagnóstica para detectar y tratar los cambios patológicos que ocurren durante la fase aguda del periodo postraumático.

En la actualidad la practica de TAC secuencial en los pacientes con TCG es una rutina clínica, pero no existe aún consenso sobre el número y la frecuencia de los controles que deben ser realizados, y esto es particularmente cierto en los pacientes que muestran lesiones Tipos I-II en la TAC inicial. Mientras algunos autores afirman que la evaluación neurológica periódica y la medición de la presión intracraneal (PIC) bastan para detectar la aparición de nuevas lesiones focales, o de efecto masa generalizado (swelling cerebral) en estos enfermos ${ }^{23}$, otros opinan que la exploración neurológica, artefactada siempre por la sedorrelajación, e incluso la monitorización de la PIC, son menos eficaces que el examen TAC secuencial protocolizado para diagnosticar precozmente lesiones masa potencialmente quirúrgicas, o swelling cerebral generalizado que requiera tratamiento médico o craniectomía descompresiva ${ }^{15,26}$.

Aunque algunos autores consideran improcedente la realización de TACs de control a intervalos fijos y los practican sólo cuando ocurre empeoramiento clínico o elevación de la PIC, es indudable que con frecuencia el deterioro neurológico y/o la elevación de la PIC se detectan demoradamente respecto al desarrollo del efecto masa, lo que puede resultar en la producción de daño neurológico adicional que a veces, y aún actuando con rapidez, resulta irreversible $\mathrm{e}^{1,2,7,9,13-16,21,27,29}$. En cualquier caso, tanto la TAC secuencial, como la medición de la PIC, aplicadas aislada o conjuntamente, parecen superar al examen clínico para detectar la aparición de nuevo efecto masa en pacientes en los que la imprescindible sedorrelajación limita o impide un seguimiento clínico fiable. Sin embargo, la utilidad diagnóstica comparativa de estos dos métodos de seguimiento está por determinar. Además, esta comparación es dificil de realizar porque uno de los métodos proporciona una información continua, en tanto que el otro aporta visiones patológicas fijas a intervalos de tiempo variables. Para complicar más el análisis no se debe olvidar que en la práctica diaria se registran tanto elevaciones de la PIC, como cambios patológicos en la TAC que carecen de verdadera significación clínica al no requerir ninguna medida terapéutica especifica.

Con el objetivo de determinar cual de los dos métodos de monitorización del incremento de volumen intracraneal i.e., la monitorización de la PIC y el examen TAC secuencial es más eficaz para detectar la aparición de nuevo efecto masa focal o generalizado durante el periodo agudo postraumático y determinar el grado de concordancia entre los hallazgos con ambas técnicas, se diseñó este estudio prospectivo en el que se monitorizó la PIC desde el inicio del curso y se practicó TAC secuencial en una serie de pacientes con TCG que mostraron lesiones difusas tipos I y II en la TAC de admisión.

\section{Material y métodos}

Entre Enero del 2001 y Diciembre del 2002 fueron admitidos en la Unidad de Politrauma un total de 56 pacientes con TCG, definido como aquél que conlleva una puntuación de 8 o menor en la Escala de Coma de Glasgow $(\mathrm{ECG})^{30}$ tras resucitación o deterioro a este "score" dentro de las primeras 6 horas después del trauma, y que además cumplieron los siguientes criterios de inclusión: edad entre 15 y 80 años, mostrar lesiones Tipo I-II en la TAC de admisión practicada dentro de las primeras 24 horas tras el impacto, y haber sido sometidos a monitorización de la PIC. Se excluyeron los pacientes con criterios de muerte cerebral al ingreso. La mayoría de los pacientes fueron enviados desde el lugar del accidente y los restantes desde hospitales regionales. Todos los traslados se hicieron en UCI móvil o helicóptero.

Los pacientes fueron manejados de acuerdo a un protocolo diagnóstico (multimonitorización y TAC secuencial) y terapéutico médico-quirúrgico preestablecido ${ }^{14,24}$. A menos que la situación hemodinámica y/o respiratoria lo impidiera, la práctica de TAC secuencial se planificó a las 12 horas tras el trauma si el inicial fue obtenido en $<3-4$ horas, 
y 24, 48 y 72 horas tras el impacto, realizándose además otros adicionales siempre que concurriera elevación no explicable de la PIC, o deterioro clínico inesperado.

La monitorización de la PIC, que se llevó a cabo con sensor intraparenquimatoso (Camino), se instauró tras realizar el TAC de admisión, y se mantuvo por periodos variables según la evolución clínica, (media $=7,9$ días) los hallazgos en la TAC de control, y la tendencia del propio parámetro.

Las variables recogidas de manera prospectiva que fueron introducidas en un banco de datos fueron las siguientes:

Epidemiológicas y clínicas. Edad y sexo, patología previa al trauma, mecanismo del trauma, existencia de politrauma (facial, ortopédico, torácico, abdominal, raquídeo, y de partes blandas), existencia de parada cardiorrespiratoria o hipotensión-hipoxemia peritraumáticas ( $\mathrm{TA}<90 \mathrm{mmHg}$ y $\mathrm{pO}_{2}<60 \mathrm{mmHg}$ ), alteraciones hematológicas como anemia (Htco $<30 \%$; $\mathrm{Hb}<10 \mathrm{~g}$ ), coagulopatía (actividad protrombina $<50 \%$; TTPA $>29$; fibrinógeno $<120$ y plaquetas $<100.000$ ), necesidad de cirugía extracraneal urgente, intervalos trauma-ingreso y trauma-TAC inicial, estado y reacción pupilar y puntuación en la ECG (bien en UCI móvil, hospital de referencia, o al ingreso en nuestro centro); ya que en la reanimación de estos pacientes se usan procedimientos y drogas que interfieren en la exploración se consideró la mejor respuesta motora en la mejor extremidad tras reducir la sedación.

Variables radiológicas. Se consideraron los siguientes hallazgos en la TAC inicial: hemorragias petequiales, hemorragia subaracnoidea (HSA), hemorragia intraventricular (HIV), contusión cerebral y/o hematoma extraaxial < 20-25 cc. En los TAC de control se constató la aparición de nuevas lesiones con efecto masa focal, o el crecimiento de lesiones preexistentes extra o intraxiales, y la aparición de swelling cerebral generalizado (lesión tipo III) o desviación de línea media.

Variables tratamiento y evolución final. Se consideraron la necesidad de indicación quirúrgica establecida por presencia de lesiones $>25 \mathrm{cc}$ en la TAC control, o de craniectomía descompresiva cuando la PIC no fuera controlable médicamente, el tipo de lesión evacuada (contusión, hematoma epidural), el número de veces que se requirió tratamiento médico especifico por PIC elevada y la presencia de complicación pulmonar y/o infecciosa. La evolución final se estimó con la Escala evolutiva de Glasgow ${ }^{8}$.

Se consideró como variable dependiente el desarrollo de deterioro definido como elevación sostenida de la PIC por encima de $20 \mathrm{mmHg}$ que requiriera tratamiento médico o quirúrgico, acompañada o no de deterioro TAC (aparición de nuevas lesiones con efecto masa, o crecimiento de lesiones preexistentes). Se consideraron los niveles de PIC (normal, moderadamente elevada e hipertensión intracraneal grave), la tendencia PIC durante el curso (baja-baja, baja-alta-baja, baja-alta, alta-baja), y la secuencia temporal o correlación entre los cambios PIC y TAC.

El análisis estadístico incluyó el estudio de la correlación entre las diferentes variables y la aparición de deterioro PIC y/o TAC por un lado, y la correlación de la elevación de la PIC y de los cambios TAC con la evolución final, por otro lado. Para ello se realizó un estudio descriptivo con cálculo de intervalos de confianza del 95\%. Para el estudio bivariable se utilizó el test del chi cuadrado, el test exacto de Fisher y la $\boldsymbol{t}$ de Student, considerándose significativos valores inferiores a 0.05 . Así mismo, se realizó estudio multivariable mediante regresión logística.

\section{Resultados}

\section{Datos epidemiológicos y clínicos}

La Tabla 1 refleja que 47 pacientes $(83,9 \%)$ fueron varones. La media de edad fue de 33,8 +/- 13,7 (DE) años. El mecanismo del trauma fue atropello en el $8,9 \%$ de los casos, accidente de automóvil en $51,8 \%$, accidente de moto en $21,4 \%$, caída en $16,1 \%$ y agresión (impacto directo) en $1,8 \%$.

46 pacientes $(84,2 \%)$ fueron trasladados directamente desde el lugar del accidente, y el 15,8\% restante desde otro hospital. El intervalo medio entre trauma e ingreso fue de 80,6 minutos y el transcurrido entre trauma y TAC inicial de 150,4 minutos. El intervalo entre el TAC inicial y el primer control fue de $10+/-7,9$ horas, y un $89,2 \%$ de los pacientes tuvieron el primer control TAC dentro de las primeras 24 horas después del trauma.

44 pacientes $(78,6 \%)$ presentaron lesiones extracraneales asociadas, lo que indica la importancia del traumatismo. 27 pacientes $(47,3 \%)$ presentaron trauma torácico asociado; $40,3 \%$ trauma ortopédico; $29.8 \%$ trauma facial; $15,7 \%$ trauma abdominal y $14 \%$ trauma en otros lugares como partes blandas. Seis $(10.7 \%)$ pacientes precisaron de intervención quirúrgica urgente (fundamentalmente esplenectomía) antes de ser estudiados con TAC craneal. 17 pacientes $(30,4 \%)$ hipotensión arterial peritraumática, $10(17,9 \%)$ hipoxemia y $8(14,3 \%)$ presentaron ambos trastornos; por tanto un total de 25 pacientes $(44,6 \%)$ presentaron defectos en la perfusión u oxigenación cerebral más o menos marcados.

La situación neurológica (peor puntuación en la ECG dentro de las 6 primeras horas tras el impacto y reactividad pupilar) se refleja también en la Tabla 1. La mitad de los pacientes puntuaron entre 3-4, y la puntuación media fue de 5 , lo que revela la gravedad del traumatismo craneal en la serie. 12 pacientes $(21,4 \%)$ presentaban midriasis unilateral y $3(5,4 \%)$ bilateral al ingreso. 
Tabla 1

Análisis descriptivo de las variables epidemiológicas, clínicas, analíticas y radiológicas iniciales, así como de los cambios radiológicos, de la presión intracraneal y evolución clínica final en una serie de 56 pacientes con trauma craneal grave que mostraron lesiones tipo I y II en el TAC inicial

\begin{tabular}{|c|c|c|}
\hline & $\mathbf{n}$ & $\%($ IC $95 \%)$ \\
\hline \multicolumn{3}{|c|}{ Datos Epidemiológicos } \\
\hline \multicolumn{3}{|l|}{ Sexo } \\
\hline Varón & 47 & $\mathbf{8 3 , 9}(74,3-93,5)$ \\
\hline Mujer & 9 & $\mathbf{1 6 , 1}(6,5-25,7)$ \\
\hline \multicolumn{3}{|c|}{ Mecanismo del Trauma } \\
\hline Atropello & 5 & $\mathbf{8 , 9}(1,4-16,4)$ \\
\hline Coche & 29 & $\mathbf{5 1 , 8}(38,7-64,9)$ \\
\hline Moto & 12 & $\mathbf{2 1 , 4}(10,7-32,1)$ \\
\hline Agresión & 1 & $\mathbf{1 , 8}(0,1-5,2)$ \\
\hline Caída & 9 & $\mathbf{1 6 , 1}(6,5-25,7)$ \\
\hline \multicolumn{3}{|c|}{ Datos Clínicos Extraneurológicos } \\
\hline \multicolumn{3}{|c|}{ Trauma Asociado } \\
\hline $\mathrm{Si}$ & 44 & $\mathbf{7 8 , 6}(67,8-89,3)$ \\
\hline No & 12 & $\mathbf{2 1 , 4}(10,7-32,1)$ \\
\hline \multicolumn{3}{|c|}{ Intervención Extracraneal } \\
\hline $\mathrm{Si}$ & 6 & $\mathbf{1 0 , 7}(2,6-18,8)$ \\
\hline No & 50 & $\mathbf{8 9 , 3}(81,2-97,4)$ \\
\hline \multicolumn{3}{|c|}{ Hipotensión } \\
\hline $\mathrm{Si}$ & 17 & $\mathbf{3 0 , 4}(18,3-42,4)$ \\
\hline No & 39 & $\mathbf{6 9 , 6}(57,6-81,7)$ \\
\hline \multicolumn{3}{|l|}{ Hipoxia } \\
\hline $\mathrm{Si}$ & 10 & $\mathbf{1 7 , 9}(7,9-27,9)$ \\
\hline No & 46 & $\mathbf{8 2 , 1}(72,1-92,1)$ \\
\hline \multicolumn{3}{|c|}{ Hipotensión e Hipoxia } \\
\hline $\mathrm{Si}$ & 8 & $\mathbf{1 4 , 3}(5,1-23,5)$ \\
\hline No & 48 & $\mathbf{8 5 , 7}(76,5-94,9)$ \\
\hline \multicolumn{3}{|c|}{ Datos Clínicos Neurológicos } \\
\hline \multicolumn{3}{|l|}{ GCS* } \\
\hline 3 & 9 & $\mathbf{1 6 , 1}(6,5-25,7)$ \\
\hline 4 & 19 & $\mathbf{3 3 , 9}(21,5-46,3)$ \\
\hline 5 & 4 & $7,1(0,3-13,8)$ \\
\hline 6 & 9 & $\mathbf{1 6 , 1}(6,5-25,7)$ \\
\hline 7 & 14 & $25(13,7-36,3)$ \\
\hline 8 & 1 & $\mathbf{1 , 8}(0,1-5,2)$ \\
\hline \multicolumn{3}{|l|}{ Midriasis } \\
\hline No & 41 & $\mathbf{7 3 , 2}(61,6-84,8)$ \\
\hline Unilateral & 12 & $\mathbf{2 1 , 4}(10,7-32,1)$ \\
\hline Bilateral & 3 & $\mathbf{5 , 4}(0,5-11,3)$ \\
\hline
\end{tabular}




\begin{tabular}{|c|c|c|}
\hline & $\mathbf{n}$ & $\%($ IC $95 \%)$ \\
\hline \multicolumn{3}{|c|}{ Datos Analíticos } \\
\hline \multicolumn{3}{|l|}{ Anemia } \\
\hline $\mathrm{Si}$ & 9 & $\mathbf{1 6 , 1}(6,5-25,7)$ \\
\hline No & 47 & $\mathbf{8 3 , 9}(74,3-93,5)$ \\
\hline \multicolumn{3}{|c|}{ Actividad de Protrombina $<50$} \\
\hline $\mathrm{Si}$ & 4 & $7,1(0,3-13,8)$ \\
\hline No & 52 & $\mathbf{8 2 , 9}(86,2-99,7)$ \\
\hline \multicolumn{3}{|c|}{ TTPA* $>34$} \\
\hline $\mathrm{Si}$ & 6 & $\mathbf{1 0 , 7}(2,6-18,8)$ \\
\hline No & 50 & $\mathbf{8 9 , 2}(81,2-97,4)$ \\
\hline \multicolumn{3}{|c|}{ Fibrinógeno $<120$} \\
\hline $\mathrm{Si}$ & 3 & $\mathbf{5 , 4}(0,5-11,3)$ \\
\hline No & 53 & $\mathbf{9 4 , 6}(88,7-99,5)$ \\
\hline \multicolumn{3}{|c|}{ Plaquetas $<100000$} \\
\hline $\mathrm{Si}$ & 3 & $\mathbf{5 , 4}(0,5-11,3)$ \\
\hline No & 53 & $\mathbf{9 4 , 6}(88,7-99,5)$ \\
\hline \multicolumn{3}{|c|}{ Datos Radiológicos } \\
\hline \multicolumn{3}{|l|}{ TCDB* } \\
\hline Tipo I & 3 & $\mathbf{5 , 4}(0,5-11,3)$ \\
\hline Tipo II & 53 & $\mathbf{9 4 , 6}(88,7-99,5)$ \\
\hline \multicolumn{3}{|c|}{ Petequias } \\
\hline $\mathrm{Si}$ & 28 & $50(36,9-63,1)$ \\
\hline No & 28 & $50(36,9-63,1)$ \\
\hline \multicolumn{3}{|c|}{ Hemorragia subaracnoidea } \\
\hline $\mathrm{Si}$ & 37 & $\mathbf{6 6 , 1}(53,7-78,5)$ \\
\hline No & 19 & $\mathbf{3 3 , 9}(21,5-46,3)$ \\
\hline \multicolumn{3}{|c|}{ Hemorragia Intraventricular $(\mathrm{n}=54)$} \\
\hline $\mathrm{Si}$ & 19 & $\mathbf{3 3}, \mathbf{9}(21,5-46,3)$ \\
\hline No & 35 & $\mathbf{6 2 , 5}(53,7-78,5)$ \\
\hline \multicolumn{3}{|c|}{ Contusión Inicial } \\
\hline $\mathrm{Si}$ & 22 & $\mathbf{3 9 , 3}(26,5-52,1)$ \\
\hline No & 34 & $\mathbf{6 0 , 7}(47,9-73,5)$ \\
\hline \multicolumn{3}{|c|}{ Hematoma Inicial } \\
\hline $\mathrm{Si}$ & 12 & $\mathbf{2 1 , 4}(10,7-32,1)$ \\
\hline No & 44 & $\mathbf{7 8 , 6}(67,9-89,3)$ \\
\hline
\end{tabular}




\begin{tabular}{|c|c|c|}
\hline & n & $\%($ IC $95 \%)$ \\
\hline \multicolumn{3}{|l|}{ Evolución Radiológica } \\
\hline \multicolumn{3}{|l|}{ Cambio en TAC } \\
\hline $\mathrm{Si}$ & 32 & $\mathbf{5 7 , 1}(44,1-70,1)$ \\
\hline No & 24 & $\mathbf{4 2 , 9}(29,9-55,9)$ \\
\hline \multicolumn{3}{|l|}{ Nueva Contusión } \\
\hline $\mathrm{Si}$ & 15 & $\mathbf{2 6 , 8}(15,2-38,4)$ \\
\hline No & 41 & $\mathbf{7 3 , 2}(61,6-84,8)$ \\
\hline \multicolumn{3}{|l|}{ Crecimiento Contusión $(\mathrm{n}=22)$} \\
\hline $\mathrm{Si}$ & 15 & $\mathbf{6 8 , 2}(48,7-87,7)$ \\
\hline No & 7 & $\mathbf{3 1 , 8}(12,3-51,3)$ \\
\hline \multicolumn{3}{|l|}{ Nuevo Hematoma } \\
\hline $\mathrm{Si}$ & 6 & $\mathbf{1 0 , 7}(2,6-18,8)$ \\
\hline No & 50 & $\mathbf{8 9 , 2}(81,2-97,4)$ \\
\hline \multicolumn{3}{|l|}{ Crecimiento Hematoma $(n=12)$} \\
\hline $\mathrm{Si}$ & 5 & $\mathbf{4 1 , 7}(13,8-69,6)$ \\
\hline No & 7 & $\mathbf{5 8 , 3}(30,4-86,2)$ \\
\hline \multicolumn{3}{|l|}{ "Swelling" } \\
\hline $\mathrm{Si}$ & 6 & $\mathbf{1 0 , 7}(2,6-18,8)$ \\
\hline No & 50 & $\mathbf{8 9 , 3}(71,2-97,4)$ \\
\hline \multicolumn{3}{|l|}{ Evolución de la PIC } \\
\hline \multicolumn{3}{|l|}{ PIC (mmHg) } \\
\hline Normal & 8 & $\mathbf{1 4 , 3}(5,1-23,5)$ \\
\hline Elevaciones episódicas breves & 21 & $\mathbf{3 7 , 5}(24,8-50,2)$ \\
\hline Moderada & 16 & $\mathbf{2 8 , 6}(16,8-40,4)$ \\
\hline Grave & 11 & $\mathbf{1 9 , 6}(9,2-30)$ \\
\hline \multicolumn{3}{|l|}{ Tendencia PIC } \\
\hline Siempre Baja & 28 & $50(36,9-63,1)$ \\
\hline Baja-Alta-Baja & 13 & $\mathbf{2 3 , 2}(12,1-34,3)$ \\
\hline Alta-Baja & 6 & $\mathbf{1 0 , 7}(2,6-18,8)$ \\
\hline Baja- Alta & 9 & $\mathbf{1 6 , 1}(6,5-25,7)$ \\
\hline \multicolumn{3}{|l|}{ Evolución Clínica } \\
\hline Mala evolución & 20 & $\mathbf{3 5 , 7}(23,1-48,2)$ \\
\hline Buena Evolución & 36 & $\mathbf{6 4 , 2}(51,8-76,9)$ \\
\hline
\end{tabular}

* GCS ${ }^{=}$puntuación de la Escala de Coma de Glasgow

*TCDB= Clasificación según la escala radiológica del estudio del Traumatic Coma Data Bank (19)

*TTPA $=$ Tiempo Parcial de Tromboplastina Activada

Las Tablas 1 y 2 reflejan la situación hematológica tras la admisión. 9 pacientes $(16,1 \%)$ presentaron anemia. Por otra parte, $7,1 \%$ tenían una actividad de protrombina por debajo del $50 \%$ y $29,8 \%$ por debajo del $70 \%$. Seis pacientes $(10,7 \%)$ mostraron una TTPA $>$ de $34 ; 5.4 \%$ un fibrinógeno $<120$, y $5,4 \%$ cifras de plaquetas $<100.000$.
La Tabla 1 muestra la evolución final que fue: Buena recuperación en 21 (37,5\%) casos; Incapacidad moderada en $15(26,8 \%)$; Incapacidad severa en $12(21,4 \%)$ y Exitus en $8(14,3 \%)$. Así, 36 pacientes $(64,2 \%)$ tuvieron una evolución favorable, mientras que $20(35,7 \%)$ presentaron una mala evolución $(35,7 \%)$. 
Tabla 2

Datos del estudio hematológico realizado tras la admisión

\begin{tabular}{|l|c|c|}
\hline & Rango & Media (ds) \\
\hline Hematocrito & $21-49$ & $37,8(+/-6,9)$ \\
\hline Hemoglobina & $7-17$ & $12,9(+/-2,3)$ \\
\hline Actividad Protrombina & $37-109$ & $79,2(+/-17,6)$ \\
\hline Fibrinógeno & $63-316$ & $194,2(+/-57,2)$ \\
\hline Tiempo de Cefalina & $12-46$ & $29,7(+/-5,6)$ \\
\hline Plaquetas & $83000-477000$ & $206685,2(+/-65546,7)$ \\
\hline
\end{tabular}

Tabla 3

Análisis de la correlación entre el desarrollo de deterioro, definido como elevación sostenida de la presión intracraneal, y diferentes variables

\begin{tabular}{|c|c|c|c|c|}
\hline & \multicolumn{4}{|c|}{ DETERIORO } \\
\hline & Si & No & OR (IC95\%) & $\mathbf{p}$ \\
\hline \multicolumn{5}{|l|}{ Contusión Inicial } \\
\hline $\mathrm{Si}$ & 15 & 7 & $\mathbf{3 , 9}(1,26-12,3)$ & \multirow{2}{*}{$\mathbf{0 , 0 1}$} \\
\hline No & 12 & 22 & & \\
\hline \multicolumn{5}{|l|}{ Cambio TAC } \\
\hline $\mathrm{Si}$ & 21 & 11 & $\mathbf{5 , 7}(1,8-18,6)$ & \multirow{2}{*}{0,003} \\
\hline No & 6 & 18 & & \\
\hline \multicolumn{5}{|l|}{ Crecimiento contusión } \\
\hline $\mathrm{Si}$ & 8 & 5 & $\mathbf{0 , 5}(0,07-3,1)$ & \multirow{2}{*}{0,6} \\
\hline No & 7 & 2 & & \\
\hline \multicolumn{5}{|l|}{ Crecimiento Hematoma } \\
\hline $\mathrm{Si}$ & 2 & 2 & $\mathbf{0 , 3}(0,03-4,2)$ & \multirow{2}{*}{0,5} \\
\hline No & 6 & 2 & & \\
\hline \multicolumn{5}{|l|}{ "Swelling" } \\
\hline $\mathrm{Si}$ & 6 & 0 & 62,8 & \multirow{2}{*}{0,007} \\
\hline No & 21 & 29 & & \\
\hline
\end{tabular}

\section{Descripción de los hallazgos TAC inicial-secuencial}

TACS iniciales. La Tabla 1 refleja la incidencia de los diferentes hallazgos patológicos observados en este tipo de pacientes; la mitad mostraron petequias visibles en la sustancia blanca subcortical, ganglios basales, cuerpo calloso o tronco cerebral, 66,1\% HSA y $33,9 \%$ HIV. 22 pacientes $(39,3 \%)$ presentaron focos contusivos y 12 $(21,4 \%)$ hematomas extra-axiales epi o subdurales siempre $<25$ cc.

TACs secuenciales. La Tabla 1 refleja los cambios patológicos observados durante la primera semana del curso postraumático. Un total de $32(57,1 \%)$ pacientes mostraron cambios en las TACs de control, la mayoría de los cuales fueron objetivados dentro de los cuatro primeros días del curso, y que consistieron en aparición de nuevos focos contusivos ( $26,8 \%$ de los casos), crecimiento de focos contusivos existentes en la TAC de admisión (68,2\%), (Fig. 1) desarrollo de nuevo hematoma extraaxial $(10,7 \%)$, o crecimiento de uno previamente existente $(41,7 \%)$, y swelling cerebral generalizado $(10,7 \%)$.

Un total de $23(41,1 \%)$ pacientes desarrollaron complicaciones infecciosas, casi siempre de asiento pulmonar que pudieron contribuir a una evolución adversa en al menos $10(17,8 \%)$ de ellos que presentaron síndrome de distress respiratorio y swelling cerebral generalizado.

Descripción de los cambios en los niveles de PIC 

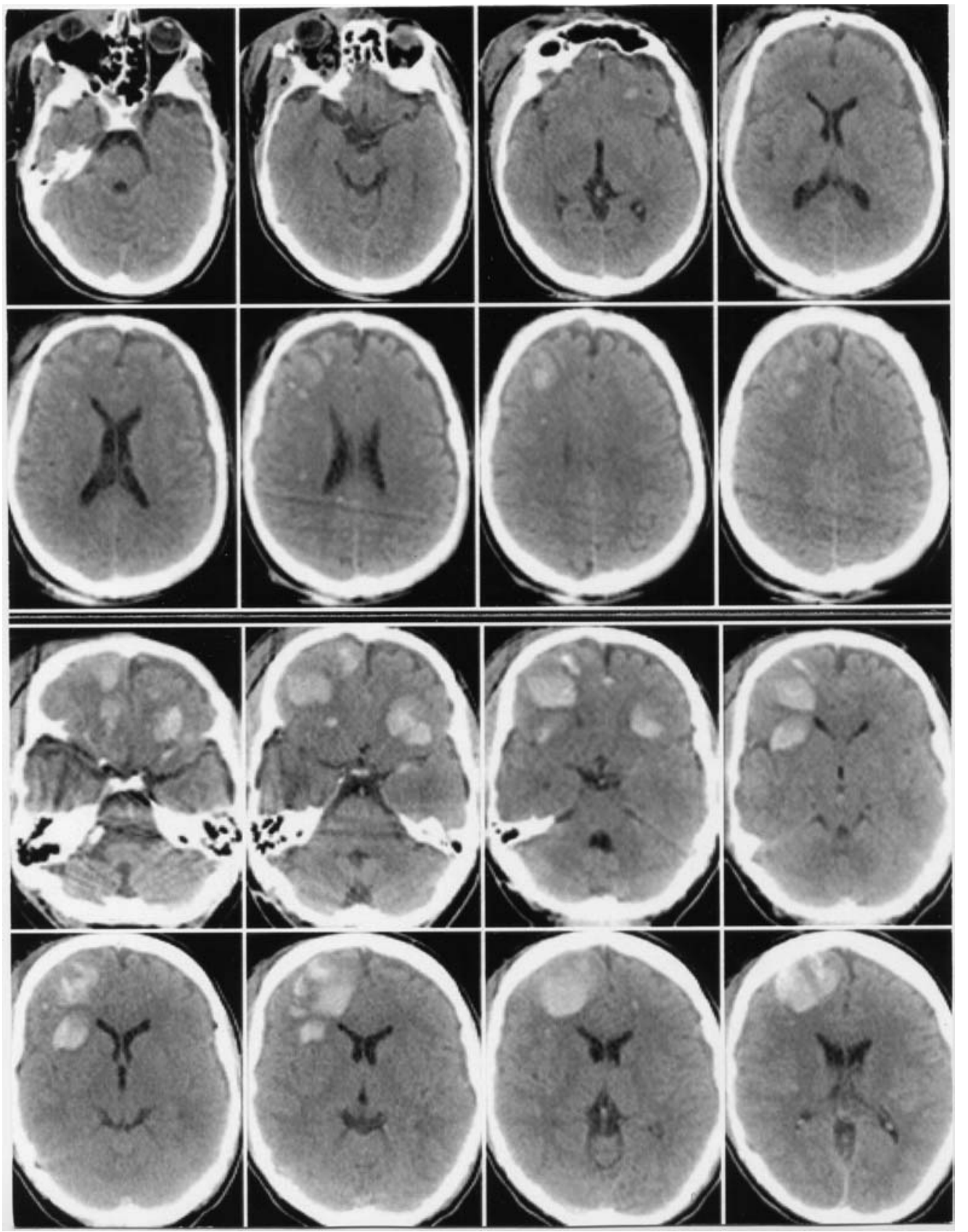

que aumentaron de volumen en un intervalo de 7 horas (figuras inferiores). La PIC permaneció dentro de límites normales y el paciente evolucionó bien sin necesidad de cirugía.

Figura 1. Paciente de 22 años que sufre impacto craneal en accidente de tráfico. Hipotensión transitoria en el lugar del accidente que se remonta. Puntuaba 7 en la ECG y la reactividad pupilar era normal. No presentaba trauma extracraneal, cambios hemodinámicos o gasométricos, ni alteraciones en el estudio de coagulación. La TAC inicial (figuras superiores) practicada 6 horas tras el impacto mostró pequeños focos contusivos en el lóbulo frontal izquierdo

La Tabla 1 muestra los niveles absolutos y el curso de la PIC durante el curso agudo postraumático. 8 pacientes $(14,3 \%)$ mostraron una PIC normal y un registro plano; $21(37,5 \%)$ desarrollaron elevaciones fásicas (duración < 15 minutos) sobre un nivel de PIC basal normal relacionadas o no con maniobras de limpieza-aspiración bronquial, cambio postural u otros factores, que con frecuencia precisaron de terapia médica no sostenida o agresiva. Por tanto, se puede considerar que $29(51,7 \%)$ pacientes no desarrollaron hipertensión intracraneal a lo largo del curso agudo postraumático. 16 pacientes $(28,6 \%)$ mostraron ele- vaciones mantenidas de duración limitada, pero que requirieron tratamiento médico repetido, por lo que se consideró que estos sufrieron hipertensión intracraneal moderada. Finalmente, 11 (19,6\%) pacientes presentaron hipertensión intracraneal grave con cambio en la tendencia del registro a elevación sostenida de la PIC; todos ellos precisaron tratamiento agresivo de la PIC que pudo ser finalmente controlada en $4(7,1 \%)$ y que resultó incontrolable y fatal en los otros $7(12,5 \%)$. El número medio de tratamientos médicos por hipertensión intracraneal en los 27 pacientes fue 13.5 +/- 15,9 (DE); rango 0-82 tratamientos. La Tabla 
Tabla 4

Análisis de la correlación entre la presencia de hipertensión intracraneal (HIC) y la evolución clínica final a incapacidad severa o muerte

\begin{tabular}{|c|c|c|c|c|c|c|c|}
\hline & & \multicolumn{3}{|c|}{ MUERTE } & \multicolumn{3}{|c|}{ INCAPACIDAD GRAVE } \\
\hline & & $\mathbf{S i}$ & No & Total & $\mathbf{S i}$ & No & Total \\
\hline & & \multicolumn{3}{|c|}{ Casos $n^{\circ}(\%)$} & \multicolumn{3}{|c|}{ Casos $n^{\circ}(\%)$} \\
\hline \multirow[t]{4}{*}{ HIC } & Si & $7(25,9)$ & $20(74,1)$ & 27 & $3(11,1)$ & $24(88,9)$ & 27 \\
\hline & No & $1(3,4)$ & $28(96,6)$ & 29 & $9(31)$ & $20(69)$ & 29 \\
\hline & TOTAL & $8(14,3)$ & $48(85,7)$ & $56(100)$ & $12(21,4)$ & $44(78,6)$ & $56(100)$ \\
\hline & & \multicolumn{3}{|l|}{$p=0,02$} & \multicolumn{3}{|c|}{ OR $(\mathbf{I C 9 5 \%})=\mathbf{0 , 2 8}(0,07-1,17)$} \\
\hline
\end{tabular}

Tabla 5

Análisis estratificado de la evolución clínica final según la presencia de hipertensión intracraneal (HIC) y cambios en el TAC

\begin{tabular}{|l|l|l|l|l|c|c|}
\hline $\begin{array}{l}\text { Cambio } \\
\text { PIC (HIC) }\end{array}$ & $\begin{array}{l}\text { Cambio } \\
\text { TAC }\end{array}$ & $\begin{array}{l}\text { Buena } \\
\text { Evolución }\end{array}$ & $\begin{array}{l}\text { Incapacidad } \\
\text { grave }\end{array}$ & Muerte & TOTAL & Cirugía \\
\hline \multicolumn{7}{|c|}{ Casos n' (\%) } \\
\hline \multirow{3}{*}{ Si } & Si & $12(21,4)$ & $3(5,4)$ & $6(10,7)$ & $21(37,5)$ & $8(14,3)$ \\
& No & 58,9 & 0 & $1(1,8)$ & $6(10,7)$ & - \\
\cline { 2 - 8 } & Total & $17(30,4)$ & $3(5,4)$ & $7(12,5)$ & $27(48,2)$ & $8(14,3)$ \\
\hline \multirow{3}{*}{ No } & Si & $5(8,9)$ & $5(8,9)$ & $1(1,8)$ & $11(19,6)$ & - \\
& No & $14(14,6)$ & $4(7,1)$ & $(0)$ & 1832,1 & - \\
\cline { 2 - 8 } TOTAL & Total & $19(33,9)$ & $9(16,01)$ & $1(1,8)$ & $29(51,8)$ & - \\
\hline
\end{tabular}

*HIC = Hipertensión intracraneal

*Buena Evolución = Buena recuperación e incapacidad moderada

1 muestra también las tendencias o patrones del parámetro PIC durante el periodo agudo postraumático. Sólo la mitad de los pacientes mostraron una PIC que se pudo considerar siempre baja.

\section{Correlación entre diferentes variables clínico-patológicas y aparición de deterioro}

La Tabla 3 muestra que del total de variables clínico-epidemiológicas, analíticas y patológicas, sólo la presencia de contusión en la TAC inicial $(\mathrm{p}=0,01)$ y la aparición de cambio TAC $(\mathrm{p}=0,003)$ se correlacionaron significativamente con el desarrollo de deterioro. Sin embargo, de los posibles cambios TAC observados, únicamente el swelling cerebral generalizado se correlacionó fuertemente con el riesgo de deterioro $(p=0,007)$ no alcanzando, por el contrario, significación estadística el crecimiento de contusión $(\mathrm{p}=0,6)$, o de hematoma extraaxial $(p=0,5)$ previamente existentes. En el estudio multivariable sólo la presencia de contusión inicial y el desarrollo de swelling cerebral se revelaron como factores asociados con deterioro.

\section{Correlación entre el desarrollo de deterioro y evolución final}

La Tabla 4 muestra la correlación entre desarrollo de deterioro (hipertensión intracraneal) y la evolución a incapacidad severa y muerte. La presencia de HIC incrementó casi diez veces $(\mathrm{OR}=9,8)$ el riesgo de muerte y de los 8 pacientes que fallecieron sólo uno dejó de presentar este cambio. Por el contrario este factor no influyó en el riesgo de desarrollar incapacidad severa, e incluso pareció tener un efecto protector $(\mathrm{OR}=0,28)$ que podría ser debido a la ausencia en este subgrupo de los pacientes fallecidos por haber presentado HIC finalmente incontrolable. 


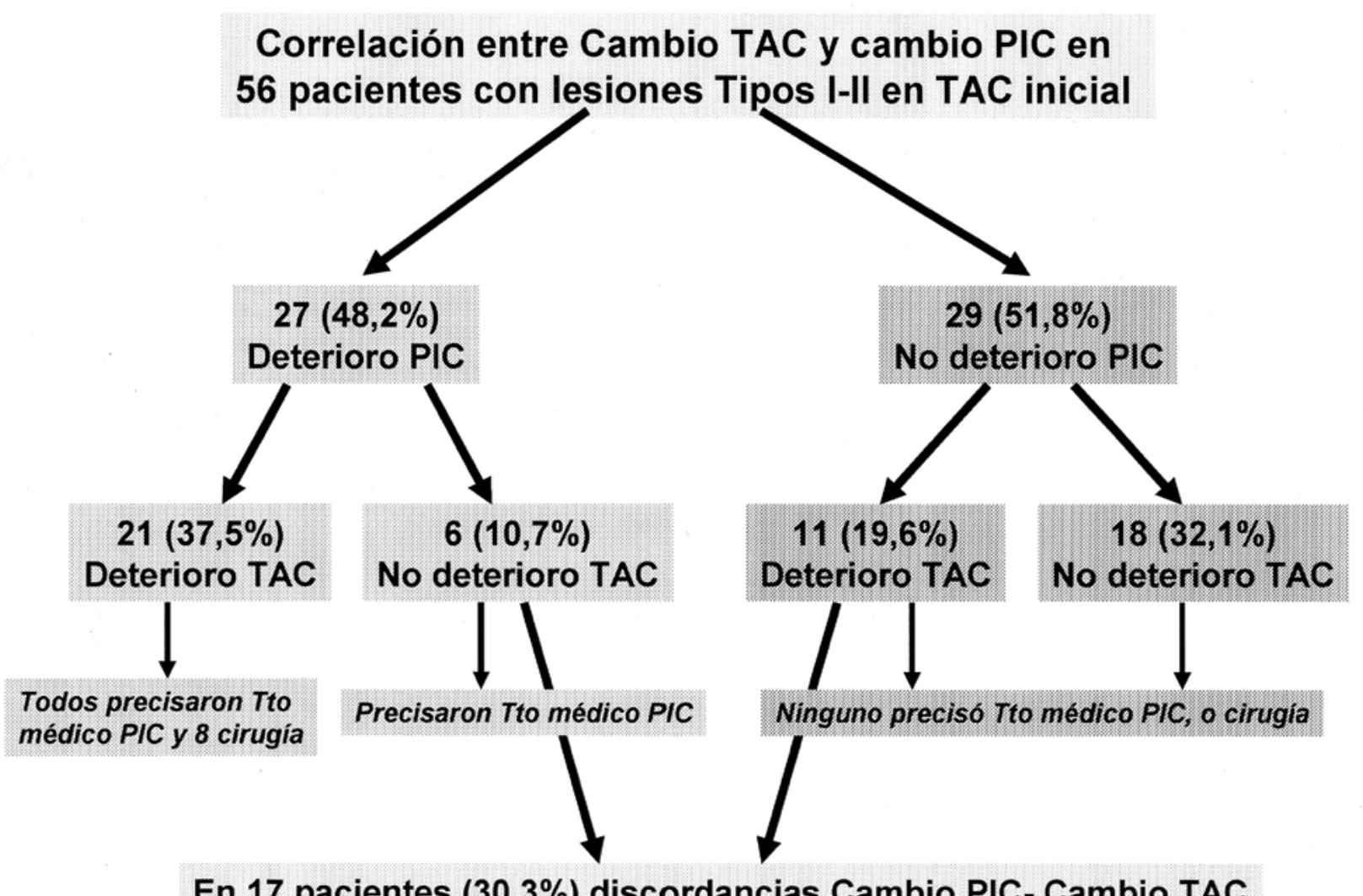

Figura 2. Diagrama de flujo de los cambios en la TAC secuencial y en la PIC, y el tipo de tratamiento aplicado en 56 pacientes con lesiones Tipo I-II en la TAC inicial.

Si bien parece indudable que la hipertensión intracraneal grave incrementa significativamente el riesgo de muerte, la escasez de la muestra en la serie no permite determinar la contribución del nuevo efecto masa y/o la elevación de la PIC al desarrollo de incapacidad moderada y grave en los pacientes que no fallecieron, que parecen fundamentalmente debidas a la lesión axonal difusa.

Correlación o interrelación de los cambios en la TAC secuencial y los cambios en la PIC

La Tabla 5 y la Figura 2 muestran que 27 pacientes $(48,2 \%)$ de los 56 de esta serie desarrollaron deterioro definido como elevación sostenida de la PIC; de estos 21 (37,5\%) mostraron cambios concurrentes en la TAC, en tanto que $6(10,7 \%)$ no mostraron nuevas patologías.

Los restantes 29 pacientes $(51,7 \%)$ no presentaron deterioro de la PIC, y de ellos $11(19,6 \%)$ mostraron cambios concurrentes en la TAC, mientras que $18(32,1 \%)$ no lo hicieron.

Globalmente, un total de $11(19,6 \%)$ pacientes mostraron cambios TAC sin cambios relevantes en la PIC, mientras que $6(10,7 \%)$ desarrollaron hipertensión intracraneal sin que la TAC de control revelara nuevas lesiones focales o difusas, o cambios de las preexistentes que pudieran explicarlos de algún modo, lo que arroja un $30,3 \%$ de discordancias entre ambos tipos de cambio.

Como se puede apreciar los 8 pacientes que precisaron cirugía (contusión cerebral en 5, hematoma extra-axial en 2, y/o craniectomía descompresiva en 3 casos), habían presentado hipertensión intracraneal acompañada por cambio en la TAC de control, si bien en los restantes 13 con ambos tipos de cambio la elevación de la PIC pude ser manejada con tratamiento médico. Por otra parte, ningún paciente sin hipertensión intracraneal precisó cirugía, independientemente de que presentara (11 casos), o no presentara (18 casos) cambio en la TAC secuencial y, como ya se ha dicho, sólo uno de ellos falleció. Independientemente del nivel de PIC, los pacientes que desarrollaron cambios en la TAC evolucionaron peor que aquellos que no los presentaron, pero la diferencia no fue estadísticamente significativa.

\section{Discusión}

Múltiples estudios realizados en la era TAC han demostrado que un gran número de pacientes con TCG desarrollan 
lesiones demoradas con efecto masa focal o generalizado como son nuevos hematomas extra o intraparenquimatosos, o swelling cerebral generalizado, que muchas veces se acompañan de deterioro clínico y empeoran la evolución final, por lo que algunos autores han tratado de identificar los factores de riesgo para desarrollar nuevo efecto masa intracraneal, así como el "timing" de su aparición durante el periodo agudo postraumático, con el objetivo principal de diseñar la mejor estrategia para el manejo diagnóstico y terapéutico de estos pacientes ${ }^{1-5,7,9-11,13,15-17,21-23,25-29}$ Sin embargo, sigue sin resolverse el debate sobre cual debe ser la metodología para la práctica de TAC secuencial y la monitorización de la PIC en los pacientes con TCG que muestran, o bien un TAC inicial normal, o hallazgos patológicos sin efecto masa relevante, (lesiones tipos I-II) y en qué medida determinados parámetros epidemiológicos, clínicos o los cambios en la evolución neurológica pueden modificar el empleo de estas técnicas de monitorización.

Al comentar los estudios publicados en la literatura relacionados con este problema clínico, y siguiendo un orden temporal analizaremos en primer lugar el de Roberson y cols ${ }^{23}$, que compararon el valor de la TAC secuencial con el examen neurológico y los cambios en la PIC en una serie de 95 pacientes con TCG. 38 de ellos (40\%) tenían una TAC normal, o casi normal al ingreso, y 80\% evolucionaron favorablemente. Sin embargo, 7 (18\%) de los 38 pacientes experimentaron cambios patológicos apreciables en la TAC de control, si bien ninguno de ellos precisó de cirugía. Según estos autores "en ningún caso la TAC programada a intervalos fijos reveló lesiones quirúrgicas que no hubieran sido sospechadas antes por los cambios ocurridos por la exploración neurológica o los niveles de PIC", por lo que conceden a la TAC de control un valor "confirmatorio" de lo anunciado por los cambios clínicos y de la PIC, y concluyen que a pesar de todo "es útil obtener TAC de control entre 3 y 7 días después del trauma si el paciente no mejora".

En relación con este estudio resulta difícil aceptar que en verdad casi la mitad de los enfermos tuvieran una TAC normal, o casi normal en el momento de la admisión, por lo que se deben sospechar, o bien la existencia de sesgos no aclarados en la selección de los pacientes, o bien una interpretación inadecuada de los TAC, a lo que pudiera haber contribuido que el estudio se realizó con máquinas de primera-segunda generación. En cualquier caso, y como se desprende de los resultados de estudios que se comentan mas adelante, en la actualidad resulta inaceptable preconizar la práctica de TAC de control entre el tercer y el séptimo días del periodo agudo postraumáticos en todos los enfermos con TCG sin matizar la indicación en función de los hallazgos en la TAC inicial, o el intervalo trauma-TAC inicial.

Kobayashi y cols ${ }^{10}$, analizaron la aparición de nuevas lesiones con TAC secuencial en una serie de 138 pacientes con TCG; 91 de ellos mostraron nuevos hallazgos en la TAC de control, que en 60 eran lesiones relevantes consistentes en edema, infarto o hematoma intra o extracerebral; estos últimos pacientes experimentaron una evolución significativamente peor que los restantes 78 que no mostraron nuevas lesiones. En esta serie, 78 (56.5\%) de los 138 pacientes tenían lesiones que hubieran sido clasificadas como difusas en la TAC inicial, pero no se analizaron específicamente los hallazgos en este subgrupo, por lo que desconocemos cual fue la incidencia de nuevas lesiones en el mismo. Por otro lado, este estudio, como el anterior, es previo a la descripción de la clasificación del TCDB, por lo que no se puede determinar el porcentaje de pacientes incluidos con lesiones tipos I-II. Aunque no se monitorizó rutinariamente la PIC y los autores no describen los cambios encontrados, concluyen que este parámetro puede servir de guía para indicar la práctica de TAC secuencial.

Lee y Liu ${ }^{13}$, analizaron la correlación entre el descenso del score en la ECG y el deterioro en la TAC secuencial en una serie de 117 pacientes con trauma moderado y grave. En el grupo de enfermos en los que el score no cambió o subió, 73\% mostraron mejoría en la TAC; por el contrario, $78 \%$ de los que presentaron deterioro clínico subsiguiente (descenso en el score) mostraron empeoramiento en la TAC de control. Estos autores refieren que 17\% de sus enfermos desarrollaron complicaciones durante el traslado a la sala TAC, que incluyeron subidas de PIC, desaturación, o cambios hemodinámicos, por lo que recomiendan practicar TAC de control sólo si ocurre deterioro clínico, cuando no hay posibilidad de llevar a cabo el examen neurológico, o si la subida de la PIC no se puede explicar por problemas médicos. De acuerdo con Teasdale, es criticable en este estudio que 19 de 60 enfermos que mostraron nuevo hematoma extraaxial en la TAC de control no fueran intervenidos, y que otros 41 en los que se objetivó un incremento de efecto masa o edema adicional, tampoco recibieran un tratamiento específico. En cualquier caso, en este estudio no se consideran separadamente, o en detalle, los hallazgos en los pacientes con lesiones difusas tipos I y II, por la misma razón que en los anteriores, y tampoco se propugna una estrategia concreta para la practica de TAC secuencial.

Lobato y cols $^{17}$, en un estudio retrospectivo sobre 448 pacientes con TCG observaron que los 46 (10\%) que tuvieron una TAC normal durante todo el periodo agudo postraumático no experimentaron elevaciones mantenidas de la PIC que requirieran tratamiento específico, y en todos ellos la evolución final fue favorable. Sin embargo, los autores enfatizan que estos 46 pacientes representaron sólo dos tercios del total de 69 que habían presentado una TAC inicial normal o casi normal, indicando que uno de cada tres pacientes con TAC inicial normal (un 5.2\% del total de la serie) desarrollaron nuevas patologías en la TAC de 
control que cursaron con hipertensión intracraneal y precisaron manejo terapéutico adicional. Aunque en el estudio se afirma que la monitorización de la PIC puede ser omitida "cuando el paciente presenta una TAC normal" los autores reconocen que no se puede excluir con seguridad absoluta a ningún paciente con TAC de admisión normal del beneficio que puede derivarse de dicha monitorización ya que existe una probabilidad sobre tres de que aparezca nuevo efecto masa intracraneal; por ello el estudio concluye recomendando una estrategia combinada que puede incluir indicación rutinaria de medición de la PIC en todos los casos y/o practica de TAC de control a intervalos cortos y prefijados, salvo si el paciente experimenta una mejoría rápida tras el ingreso.

En el estudio sobre 753 pacientes con trauma grave del TCDB americano, Eisenberg y $\operatorname{cols}^{6}$, encontraron que un $10-15 \%$ de los pacientes con TAC normal en el momento de la admisión desarrollaron elevaciones de la PIC durante el periodo agudo postraumático. La razón por la que la incidencia de hipertensión intracraneal en pacientes con TAC inicial normal fue 2 a 3 veces mas alta que en el estudio mencionado de Lobato y cols $^{17}$, queda por determinar, si bien una posibilidad es que la definición de hipertensión intracraneal o la calidad de los estudios TAC fueran distintas en ambos estudios.

En otro estudio posterior de Lobato y cols ${ }^{15}$, sobre TAC secuencial en pacientes con TCG, un total de 271 (30.3\%) mostraron lesiones difusas, bien tipo I ( 24 casos) o bien tipo II (247 casos) en la TAC de admisión. 4 (16,6\%) de los 24 pacientes con lesión tipo I desarrollaron nuevo efecto masa focal o difuso que precisó tratamiento específico, porcentaje este similar al referido por Eisenberg y cols ${ }^{6}$. Por otra parte, de los 247 pacientes con lesión tipo II, 31 $(12.5 \%)$ y $19(7.6 \%)$ desarrollaron nueva masa focal o efecto masa difuso, respectivamente que requirió así mismo tratamiento. Por tanto, un $20 \%$ de los pacientes ( 1 de cada 5) con lesiones tipos I-II en la TAC inicial desarrollaron nuevo efecto masa intracraneal. La evolución final fue favorable en el $82 \%$ de los pacientes con lesión tipo I que no desarrollaron cambios, frente a tan sólo el $56 \%$ en los que el TAC demostró nuevo efecto masa, $(\mathrm{p}<0.05)$; en los pacientes con lesión tipo II la evolución fue favorable en el $66 \%$ de los que no presentaron cambios, en comparación con el 36\% de los que desarrollaron cambios en la TAC; $(p<0.01)$. Los cambios patológicos ocurrieron en su mayor parte en los dos primeros días después del impacto, por lo que los autores recomiendan practicar al menos un TAC de control en este intervalo de tiempo. La práctica totalidad de las nuevas lesiones con efecto masa relevante se acompañaron de elevación de la PIC, pero este parámetro no se monitorizó desde el inicio en todos los casos y tampoco se analizó la correlación entre el "timing" de los cambios PIC y los cambios TAC.
En un estudio más reciente, Servadei y $\operatorname{cols}^{26}$, determinaron la aparición de nuevas lesiones intracraneales durante el periodo agudo postraumático en la serie de pacientes con trauma grave y moderado recogida prospectivamente y recomiendan igualmente practicar TAC secuencial en los pacientes que muestran inicialmente lesiones difusas, ya que uno de cada 6 tiene riesgo de desarrollar cambios patológicos clínicamente relevantes. En efecto, de 464 pacientes inicialmente etiquetados de haber sufrido una lesión cerebral difusa, 76 (16\%) mostraron cambios patológicos relevantes en la TAC de control. De un total de 80 pacientes con lesión inicial tipo I, 6 evolucionaron a lesiones tipos II-III y 4 a masa evacuable; de los 219 inicialmente diagnosticados de lesiones difusas tipo II, 13 evolucionaron a lesiones tipos III-IV, y 36 a masa evacuable. Al igual que en el estudio de Lobato y cols, el desarrollo de masa focal se asoció a una evolución final significativamente más desfavorable, (62\% vs $38 \%$ de evolución adversa con y sin cambio patológico, respectivamente; $(\mathrm{p}<0.01)$, pero tampoco se analizó la correlación entre los cambios en la PIC y las variaciones en la TAC secuencial.

En un estudio previo Servadei y $\operatorname{cols}^{27}$, encontraron que 15 de un total de 37 pacientes con lesiones masa detectadas con TAC de control practicado dentro de las primeras 12 horas tras el trauma, habían mostrado lesiones difusas en la TAC inicial (2 tipo I, 7 tipo II y 6 tipos III-IV). Las nuevas lesiones masa en los 9 enfermos con lesiones iniciales tipos I y II fueron detectadas bien por deterioro clínico, o bien mediante TAC de control programado, y cuando el TAC se obtiene dentro de las 3 primeras horas después del impacto los autores recomiendan repetirlo dentro de las siguientes 12 horas. En un comentario a este artículo Chesnut manifiesta que la aparición de una nueva lesión, o la expansión de una previamente observada es importante no sólo para prevenir el deterioro progresivo neurológico, sino también para evitar el empleo de tratamiento médico empírico, como es el intento de reducción de la PIC elevada atribuida a una masa focal "oculta" (no visualizada con TAC e inexistente en realidad), ya que estas terapias no están exentas a su vez de riesgo. Este último autor considera interesante determinar hasta que punto el empleo de un monitor de PIC con poco riesgo como es el Camino, puede obviar la necesidad de TAC de control a intervalos fijos o programado. En otro comentario acompañante, Marshall ${ }^{18}$ señala que en cinco pacientes de esta serie la elevación de la PIC sin deterioro clínico concomitante condujo a la práctica de la TAC de control que evidenció una lesión masa a evacuar, destacando así la utilidad de la medición de este parámetro en el manejo de estos enfermos. En este sentido parece útil resaltar que no sólo es inadecuado tratar empíricamente PIC elevadas atribuidas a efecto masa no comprobado con TAC y realmente inexistente, sino que a veces se practican igualmente TACs de control superfluos sobre la base de 
una elevación "no explicada" de la PIC, que finalmente no tiene ninguna significación y es autolimitada; en esta ultima circunstancia se podría hablar de una medida PIC falsamente positiva, pero no de un manejo erróneo inducido por la información proporcionada por este método de monitorización.

Aparte de los estudios descriptivos de los cambios TAC secuenciales mencionados anteriormente, otro aspecto importante que ha ocupado a diferentes autores es el análisis de los factores asociados con el riesgo del desarrollo de lesiones demoradas. Narayan y cols ${ }^{21}$, recomendaron monitorizar la PIC en los pacientes con TCG y TAC inicial normal que tuvieran una edad $>40$ años, hipotensión peritraumática y un "score" $<5$, al observar que la incidencia de hipertensión intracraneal alcanzó el 60\% en los pacientes con dos o más de estos factores, frente a tan solo el $4 \%$ en aquellos con uno o ninguno. Aun cuando la escasez numérica de la muestra en nuestro estudio pudiera limitar la significación de los hallazgos, los únicos factores que se correlacionaron de manera estadísticamente significativa con el desarrollo de deterioro en el estudio bivariable fueron la presencia de cambio TAC, la presencia de contusión en la TAC inicial y el desarrollo de swelling cerebral generalizado, y sólo los dos últimos lo hicieron en el estudio multivariable. Así, si bien el primero de estos criterios i.e., la presencia de focos contusivos en la TAC inicial serviría para indicar monitorización de la PIC desde el inicio, la aparición de cambio TAC no serviría para establecer ni la secuencia de TAC control, ni la necesidad de monitorización de la PIC, ya que se trata de un suceso demorado en el tiempo que es en sí mismo el objeto de predicción. La presencia de marcadores de lesión axonal difusa, como son las petequias en sustancia blanca y la HIV, aún cuando se asociaron con el desarrollo de incapacidad severa, no lo hicieron con el riesgo de deterioro en la PIC. Por todo ello, y como no identificamos ningún factor o variable capaz de predecir el desarrollo de swelling cerebral generalizado, que usualmente cursa con elevación sostenida de la PIC, lo más conveniente parecería monitorizar este parámetro desde el comienzo del curso postraumático en todos los pacientes.

Otro factor considerado como posiblemente involucrado en el desarrollo de lesiones hemorrágicas demoradas son las alteraciones de la coagulación que aparecen frecuentemente tras el TCG, y que han sido analizadas en estudios tanto clínicos como experimentales ${ }^{5,9,11,25,28,29,31,32}$. Stein y cols $^{29}$, en una primera serie de 253 pacientes seleccionados para práctica de TAC secuencial por presentar un score $<7$, o no mejorar de manera rápida tras el trauma aún cuando mostraran scores mas altos, observaron que 123 (48.6\%) desarrollaron lesión intracraneal demorada; de estos $55 \%$ mostraron anormalidades en los tiempos de protrombina, tromboplastina o recuento de plaquetas en el primer estudio de coagulación, en tanto que sólo el $9 \%$ no tenían alteraciones en dichos parámetros $(\mathrm{p}<0.001)$. El estudio concluye que el riesgo de desarrollar lesión intracraneal demorada sube del $31 \%$ en ausencia del trastorno de la coagulación al $85 \%$ cuando al menos uno de los parámetros mencionados está alterado, por lo que recomiendan la práctica frecuente de TAC de control en estos pacientes.

En otro estudio subsiguiente en el que seleccionaron 337 pacientes también con trauma moderado y grave Stein y cols ${ }^{28}$, observaron que 149 (44.5\%) desarrollaron lesión intracraneal demorada que se asoció con la severidad del daño cerebral estimado inicialmente, la necesidad de resucitación cardiopulmonar en el lugar del accidente, la presencia de coagulopatía al ingreso y la de hematoma subdural en la TAC inicial. Los pacientes con lesión aparecida en la TAC de control presentaron una evolución final significativamente peor que los que no mostraron deterioro en la TAC. Aunque en ninguno de los dos trabajos mencionados se especifican los cambios ocurridos en función de la categoría diagnóstica observada en la TAC inicial, se dice que "el desarrollo de lesión cerebral demorada empeoró la evolución final en todas las categorías de lesión" y los autores recomiendan practicar TAC de rutina en el seguimiento inmediato de todos los pacientes que se deterioran, o de manera automática a las 12-24 horas tras el ingreso si hay coagulopatía, o ausencia de mejoría neurológica. Afirman que "una vez que el TAC de control evidencia la presencia de lesión cerebral adicional de aparición demorada el paciente tiene un riesgo significativamente mayor de presentar también deterioro clínico, elevación del PIC, peor evolución final y muerte", pero no consideran la posible utilidad de la TAC secuencial programada en comparación con el examen neurológico repetido, o la monitorización de la PIC. En un comentario a este trabajo Marshall aduce que la alta frecuencia de incremento volumétrico intracraneal tras la TAC inicial revela la necesidad de determinar de manera continua la presión y el volumen intracraneales mediante la monitorización de la PIC y la práctica de TAC secuencial.

Más recientemente, Oertel y cols ${ }^{22}$, analizaron la incidencia demorada de hemorragia intra o extraaxial en una cohorte de 142 pacientes con trauma craneal moderado y grave que fueron estudiados con TAC dentro de las primeras 24 horas tras el impacto; el intervalo medio traumaTAC inicial fue de 2 horas y el intervalo medio entre este examen y el primer control TAC de tan solo 7 horas. 60 (42.3\%) pacientes mostraron nueva lesión hemorrágica en la TAC de control, y en el $87 \%$ de éstos se había practicado el primer TAC dentro de las dos primeras horas tras el impacto. La probabilidad de desarrollar nueva lesión hemorrágica fue del 51\% en los pacientes que mostraron hematoma intraparenquimatoso-contusión en la TAC de admisión, $22 \%$ en los que mostraron hematoma epidural, 
$17 \%$ en los que presentaron hemorragia subaracnoidea y $11 \%$ en aquéllos con hematoma subdural. El análisis con regresión logística mostró que la edad avanzada, el sexo masculino, el TAC inicial precoz $(<2$ horas) y la prolongación del tiempo de tromboplastina fueron predictores fiables de aparición de hemorragia intracraneal demorada. Aunque se aclara que el porcentaje de pacientes con PIC $>20 \mathrm{mmHg}$ fue significativamente mayor en el subgrupo de los que desarrollaron hemorragia demorada que en el que no la presentaron, no se analizó la correlación temporal entre ambos cambios, ni en qué medida la monitorización de la PIC condicionó la secuencia de TAC de control; sin embargo, los autores apuntan que la elevación de la PIC ocurrió en un $25 \%$ de los pacientes que eventualmente necesitaron evacuación quirúrgica de su lesión hemorrágica. Dado que casi la mitad de los pacientes con trauma craneal que son estudiados con TAC dentro de las 4 primeras horas después del impacto van a desarrollar cambios hemorrágicos posteriores, Oertel y cols recomiendan repetir la TAC en un plazo de 4-6 horas después del inicial $^{22}$.

Aparte de la falta de influencia de la edad, el "score" inicial, y la existencia de hipotensión-hipoxemia peritraumáticas sobre el riesgo de deterioro observada en nuestro estudio, tampoco las alteraciones de la coagulación registradas tras el ingreso se correlacionaron con la incidencia de deterioro por un lado, el desarrollo de hematomas quirúrgicos demorados por otro, o una peor evolución final. Por tanto, y de acuerdo con nuestros hallazgos estos parámetros no sirven para diseñar la estrategia de TAC control y/o monitorización de la PIC en los pacientes con lesiones iniciales tipos I-II. La diferencia en la significación de los trastornos de la coagulación entre nuestro estudio y los de Stein y $\operatorname{cols}^{28,29}$ y Oertel y cols ${ }^{22}$ que contemplaron series más numerosas de pacientes pudiera deberse a que en ellos se incluyeron pacientes con traumatismos no solo graves, sino también moderados y pacientes con diferentes tipos de lesiones en la TAC inicial. Aunque en algunos estudios sobre hematoma intracraneal postraumático demorado no se observó una mayor incidencia de coagulopatía ${ }^{5,25}$ parece lógico pensar que este trastorno pueda favorecer la aparición de nuevas hemorragias o el incremento volumétrico de las preexistentes en los pacientes con trauma craneal ${ }^{9,11,22,31}$. y algunos estudios realizados en el laboratorio apoyan que las alteraciones de la coagulación favorecen esta patología $^{32}$. Una vez más pudiera ser que la escasez numérica de la muestra en nuestro estudio justifique las diferencias observadas con los estudios mencionados.

Teniendo en cuenta los datos que se desprenden de los estudios mencionados y del presente análisis, parece claro que los pacientes con TCG que muestran lesiones difusas en la TAC de admisión tienen un riesgo significativo de desarrollar nuevo efecto masa intracraneal que es capaz de producir deterioro neurológico o muerte, y que la mayor parte de las veces dicho cambio aparece en los dos o tres primeros días después del trauma. Sin embargo, las estrategias propuestas por los diferentes investigadores para detectar cuanto antes el incremento de efecto masa intracraneal focal o generalizado ${ }^{1,15-18,22,26-29}$, resultan dispares entre si, al apoyarse en diferente medida en el uso de los criterios clínico, patológico (TAC), y fisiológico (PIC) para practicar el seguimiento. Por consiguiente, si bien la necesidad de TAC de control y monitorización de la PIC resulta clara en los pacientes con lesiones tipos III, IV y $\mathrm{V}^{15}$, queda por determinar cual es la mejor estrategia diagnóstica en presencia de lesiones tipos I-II.

Si bien, y como ya se dijo, en el estudio de Roberson y $\operatorname{cols}^{23}$ ninguna lesión que requiriera cirugía fue detectada por TAC realizados a intervalos fijos antes de que fuera anunciada por cambios en el estado neurológico o la elevación de la PIC, no se sabe hasta que punto la monitorización de la PIC puede obviar la indicación de TACs de control preprogramados, ya que en ocasiones la hipertensión intracraneal se desarrolla bruscamente aún cuando la lesión que la motiva podría haber sido detectada previamente con la TAC y evacuada antes de causar isquemia cerebral adicional ${ }^{1}$. Para dificultar más el diseño de un algoritmo de manejo diagnóstico en este escenario clínico se ha de recordar que hay pacientes que muestran elevaciones autolimitadas de la PIC no concurrentes con cambio TAC ni deterioro clínico, mientras que otros presentan lesiones focales que si bien no se acompañan de elevación de la PIC, alcanzan un tamaño en el límite de la indicación quirúrgica, planteando la duda sobre la conveniencia de su evacuación, particularmente cuando asientan a nivel del lóbulo temporal. En cualquier caso, utilizar la aparición de deterioro neurológico como criterio guía para la práctica de la TAC de control no es aceptable, al ser precisamente el daño cerebral secundario lo que se trata de prevenir con una adecuada estrategia diagnóstica.

Así pues en la actualidad seguimos sin saber si en los enfermos que no muestran efecto masa al ingreso (aquéllos con lesiones tipos I-II) se debe practicar un TAC de control ultraprecoz seguido de controles diarios durante los 3-4 primeros días, monitorizar la PIC desde el inicio para guiar la practica de TACs de control, o recurrir al uso simultáneo de ambas estrategias diagnósticas cuya utilización conlleva algunos riesgos y los correspondientes costes.

El presente estudio, que se diseñó para tratar de responder a estas interrogantes, incluyó pacientes con lesiones tipos I-II admitidos consecutivamente tras sufrir TCG, de los que más de dos tercios tenían lesiones extracraneales asociadas, mostraban puntuaciones considerablemente bajas en la ECG, y presentaron además una incidencia elevada de hipotensión-hipoxemia peritraumáticas. Los intervalos trauma-TAC inicial y TAC inicial-primer TAC 
control fueron cortos, lo que revela un buen funcionamiento del sistema de atención extra-intrahospitalario en nuestro medio, y el número de controles TAC en la primera semana fue elevado, por lo que se puede asumir que tanto la incidencia de los cambios patológicos, como el "timing" de su aparición durante el periodo agudo postraumático, se determinaron de manera precisa. Finalmente, la PIC fue monitorizada durante periodos de tiempo suficientemente prolongados en todos los pacientes. Además, se practicaron estudios de coagulación al ingreso y controles posteriores en todos los casos. Por todo ello, se puede considerar que la serie de pacientes incluidos y la metodología del estudio son adecuados para responder a la cuestión planteada anteriormente. No obstante, una limitación del análisis podría ser la escasez numérica de la muestra que comprometería el alcance de significación estadística de algunas correlaciones entre parámetros clínicos y patológicos con el riesgo de deterioro y la evolución final.

Al analizar la evolución durante el periodo postraumático agudo lo primero que llama la atención es el elevado porcentaje de pacientes $(57,1 \%)$ que desarrollaron nuevo efecto masa focal, o swelling cerebral generalizado. Este porcentaje supera a los observados previamente por otros autores y por nosotros mismos ${ }^{15,26}$, y corrobora que el riesgo de sufrir deterioro patológico intracraneal es muy alto en estos pacientes. Sin embargo, también replica la evidencia de que una proporción importante de las lesiones encontradas no tienen significación clínica, o al menos no se acompañan de repercusión en los niveles de PIC, ni precisan ser finalmente evacuadas. Así, de los $32(57,1 \%)$ pacientes que mostraron cambios en la TAC, $11(34,3 \%)$ no desarrollaron hipertensión intracraneal (Tabla 5 y Fig. 2), y finalmente sólo $8(14,2 \%)$ en toda la serie necesitaron una intervención quirúrgica para aliviar el efecto masa. Aunque este porcentaje de lesiones operables parece relativamente bajo, si careciésemos de otro método alternativo fiable para detectarlas, deberíamos admitir que la TAC secuencial ha de ser practicada en todos los casos y a intervalos cortos, particularmente en presencia de lesión inicial tipo II, que conlleva un riesgo mas alto de desarrollar nuevo efecto masa que la lesión tipo $I^{15,22,26}$. En nuestro estudio los porcentajes de pacientes que experimentaron crecimiento de masa intra (contusión cerebral), o extraaxial (hematoma epi o subdural) visible ya en la TAC de admisión, pero lo suficientemente pequeñas para que los pacientes fueran clasificados como portadores de lesión tipo II, fueron del $68.2 \%$ y $41.7 \%$, respectivamente, aún cuando la mayor parte de ellas no tuvieran que ser finalmente evacuadas. En cualquier caso, en los pacientes que hicieron cambio TAC la monitorización de la PIC contribuyó de manera esencial a decidir cuales de ellos fueron candidatos a tratamiento médico o quirúrgico y cuales no. Por otra parte, en los 11 pacientes mencionados que desarrollaron cambio TAC sin hipertensión intracraneal, y que representan un $20 \%$ del total de casos en nuestra serie, la falta de monitorización de la PIC podría haber inducido a tratar una supuesta elevación de la misma asumida erróneamente sobre la base del cambio patológico observado en la TAC de control.

La otra observación principal en nuestro estudio fue que los pacientes con lesiones tipos I-II muestran una alta incidencia de elevación sostenida de la PIC, y así finalmente $27(48,2 \%)$ requirieron tratamiento más o menos agresivo de la hipertensión intracraneal (Tabla 5 y Fig. 2). Es importante hacer notar que en 6 de ellos $(10,7 \%)$ el cambio en la PIC no se acompañó de cambios relevantes en la TAC por lo que la utilización exclusiva de la TAC secuencial pudiera haber llevado a la omisión del tratamiento de la hipertensión intracraneal aún cuando en realidad era necesaria.

Nuestra propuesta final de manejo diagnóstico, que es la de utilizar simultáneamente los dos métodos de detección de incremento del volumen intracraneal en los pacientes con lesiones iniciales tipos I-II, se justifica también porque en un $30,3 \%$ de ellos la TAC secuencial y la monitorización de la PIC mostraron discordancias a la hora de detectar nuevo efecto masa. El hecho de que en una quinta parte $(19,6 \%)$ de los pacientes la TAC detectara efecto masa intracraneal sin que ocurriera elevación sincrónica relevante de la PIC, podría indicar que, o bien la monitorización de la PIC aportó un resultado falsamente negativo, o bien la TAC ofreció un resultado falsamente positivo, ya que en ellos la masa no precisó ser evacuada al no sobrepasar su tamaño la capacidad de compensación volumétrica intracraneal. Sin embargo, se considere o no el parámetro PIC como un falso negativo en este escenario clínico, debemos aceptar que son precisamente los enfermos que muestran inicialmente alguna patología en la TAC en los que la práctica de controles TAC secuenciales y la monitorización de la PIC parecen más claramente indicados.

La situación contrapuesta se observó en los $6(10,7 \%)$ pacientes que mostraron elevaciones de la PIC sin cambios apreciables y correlativos en la TAC de control. En esta situación podría igualmente considerarse, o bien que la PIC es un criterio diagnóstico de aumento de masa intracraneal falsamente positivo, o bien su hipótesis alternativa, es decir, que la TAC es un criterio falsamente negativo para detectar efecto masa difuso intracraneal, u otro tipo de trastorno hidrodinámico que justifique la elevación de la presión. El hecho de que en estos pacientes sin aparente patología subyacente fuera necesario tratar médicamente de manera repetida y agresiva la hipertensión intracraneal refuta que en este escenario clínico la PIC elevada represente un indicador falsamente positivo. En cambio, la consideración de falso negativo para la TAC debería ser admitida, lo que plantea cual debe ser la estrategia de control TAC en esta situación particular; las alternativas extremas serían 
o prescindir de nuevos controles TAC al observar que uno de ellos es normal, o por el contrario, acortar los intervalos de control TAC al ser casi la norma que ocurra un cambio patológico en el paciente que va a desarrollar hipertensión intracraneal. La segunda opción parece la más apropiada, ya que en algunos pacientes que desarrollan "swelling" cerebral generalizado el colapso más o menos completo de los espacios cisternales y ventriculares precede en poco tiempo a la elevación de la PIC.

La observación de elevaciones de la PIC aparentemente paradójicas que cursan sin correlato patológico reconocible, plantea la necesidad de determinar si existen pacientes en los que no es necesario tratar una PIC elevada, y si es así, cual es el perfil clinicopatológico de estos pacientes, cual el mecanismo de la hipertensión intracraneal que desarrollan, y cuales los límites de elevación y duración aceptables para obviar el tratamiento. En teoría podrían existir trastornos postraumáticos de tipo pseudotumoral en los que una alteración en la circulación-absorción del LCR podría cursar con aumento de la carga hidrostática (y por tanto elevación de la PIC) que al estar distribuida de manera homogénea en el compartimento intracraneal no colapsaría los espacios que contienen LCR, ni producirían isquemia cerebral ("pseudotumor-like conditions"). La capacidad de la TAC para demostrar alteraciones de la hidrodinámica y hemodinámica intracraneales es desde luego limitada, y parece razonable esperar que las nuevas técnicas de RM, al igual que nos están permitiendo mejorar la predicción del pronóstico final ${ }^{12}$, nos ayuden a entender el mecanismo de ciertas elevaciones de la PIC, que aunque ahora nos parecen paradójicas, pudieran relacionarse con cambios en el volumen sanguíneo y flujo cerebrales, o la distribución del agua intra-extracelular,

El hecho de que éste y otros estudios previos no hayan permitido determinar en qué medida la monitorización de la PIC permite optimizar la estrategia de TAC secuencial en los pacientes con TCG y lesiones tipo I-II en la TAC inicial, bien disminuyendo el número de controles, o por el contrario, aumentándolo en algunos enfermos, para nada limita el valor de la medición continua de la PIC en este escenario clínico. Aunque algunos autores aún sostienen que el uso combinado de monitorización de la PIC y TAC secuencial a intervalos prefijados puede resultar redundante y generar riesgos evitables y costes innecesarios, en el momento actual parece recomendable monitorizar la PIC de entrada en todos los casos. Las lesiones con efecto masa causan daño neurológico secundario cuando elevan la PIC, y es por ello que hemos utilizado la hipertensión intracraneal como criterio definitorio de deterioro, en detrimento de los hallazgos patológicos evidenciados por la TAC.

Teniendo en cuenta la evidencia proporcionada por este estudio, podría sugerirse que una buena pauta para el manejo de los pacientes comatosos (y por tanto sometidos a sedorrelajación que artefacta o elimina el valor de la exploración neurológica como criterio de seguimiento) y lesión tipo I-II en la TAC de admisión sería practicar TACs a las 4-6, 12, 24 y 48 horas tras el impacto, aumentando el numero de controles a instancias de posibles elevaciones de la PIC monitorizada desde el inicio. Es innegable que con frecuencia la TAC realizada a intervalos prefijados detecta lesiones que por su envergadura van a ir seguidas inmediatamente de deterioro PIC, permitiendo así extremar la vigilancia y acelerar el tratamiento pertinente cuando éste sea eventualmente necesario. Tomando las precauciones adecuadas es posible evitar las desaturaciones y la inestabilidad hemodinámica que pueden ocurrir durante el traslado de los pacientes a la sala TAC. Aún cuando parece indudable que detectar la presencia de nuevas masas evacuables y controlar las elevaciones de la PIC ha de conllevar una mejoría de la evolución final de estos enfermos, demostrar que la práctica de TAC secuencial y la monitorización de la PIC influyen positivamente en el resultado final requeriría un estudio prospectivo aleatorizado que no es practicable por diferentes razones, entre ellas las de tipo ético.

Aunque por definición las lesiones hemorrágicas iniciales en los enfermos con lesiones Tipo I-II son de pequeño tamaño, pudiera ser que aquéllos expuestos al riesgo de desarrollar progresión de las mismas durante el curso postraumático en la manera descrita por Oertel et $\mathrm{al}^{22}$, se beneficiaran de la administración del factor VII recombinante activado, que se ha mostrado capaz de frenar el incremento de volumen del hematoma en los pacientes con hemorragia intracerebral aguda espontánea ${ }^{20}$. De hecho, en un futuro próximo se va a emprender un estudio prospectivo aleatorizado en pacientes con lesiones hemorrágicas postraumáticas de pequeño tamaño para determinar si este factor es eficaz para limitar su crecimiento durante las primeras horas después del impacto.

\section{Bibliografía}

1. Bullock, R., Golek, J., Blake, G.: Traumatic intracerebral hematoma- which patients should undergo surgical evacuation? CT scan features and ICP monitoring as a basis for decision making. Surg Neurol 1989; 32: 181-187.

2. Clifton, G.L., Grossman, R.G., Makela, M.E., Miner, M.E., Handel, S., Sadhu, V.: Neurological course and correlated computerized tomography findings after severe closed head injury. J Neurosurg 1980; 52: 611-624

3. Cordobés, F., de la Fuente, M., Lobato, R.D. et al.: Intraventricular hemorrhage in severe head injury. J Neurosurg 1983; 58: 217-222

4. Cordobés, F., Lobato, R.D., Rivas, J.J. et al.: Posttraumatic diffuse brain axonal injury. Analysis of 78 patients studied with computed tomography. Acta Neurochir (Wien) 1986; 81: 27-35. 
5. Diaz, F.G., Yock, D.H., Larson, D., Rockswold, G.L.: Early diagnosis of delayed posttraumatic intracerebral hematomas. J Neurosurg 1979; 50: 217-223

6. Eisenberg, H.M., Gary, H.E., Aldrich, E.F., et al.: Initial CT findings in 753 patients with severe head injury. A report from the NIH Traumatic Coma Data Bank. J Neurosurg 1990; 73: 688-698.

7. Gudeman, S.K., Kishore, P.R.S., Miller, J.D., et al.: The genesis and significance of delayed traumatic intracerebral hematoma. Neurosurgery 1979; 5: 309-313.

8. Jennett, B., Bond, M.: Assessment of outcome after severe brain damage. A practical scale. Lancet 1975; 1: 480484.

9. Kaufmann, H.H., Moake, J.L., Olson, J.D., et al.: Delayed and recurrent intracranial hematomas related to disseminated intravascular clotting and fibrinolysis in head injury. Neurosurgery 1980; 7: 445-449.

10. Kobayashi, S., Nakazawa, S., Otsuka, T.: Clinical value of serial computed tomography with severe head injury. Surg Neurol 1983; 20: 25-29.

11. Kumura, E., Sato, M., Fukuda, A., et al.: Coagulation disorders following acute head injury. Acta Neurochir (Wien) 1987; 85: 23-28.

12. Lagares, A., Ramos, A., Alday, R., et al.: Valor pronóstico de la RM craneal en el traumatismo craneoencefálico moderado y grave. Patología Aparato Locomotor 2005; 3: 41 54.

13. Lee, S.T., Liu, T.N.: Delayed intracranial hemorrhage in patients with multiple trauma and shock-related hypotension. Acta Neurochir (Wien) 1991; 113: 121-124.

14. Lobato, R.D., Cordobes, F., Rivas, J.J., et al.: Outcome from severe head injury related to the type of intracraneal lesion. A computerized tomography study. J Neurosurg 1983; 59: $762-774$.

15. Lobato, R.D., Gómez, P.A., Alday, R., et al.: Sequential computerized tomography changes and related final outcome in severe head injury patients. Acta Neurochir (Wien) 1997; 139: 385-391.

16. Lobato, R.D., Rivas, J.J., Gómez, P.A., et al.: Headinjuried patients who talk and deteriorate into coma. Analysis of 211 cases studied with computerized tomography. J Neurosurg 1991; 75: 256-261.

17. Lobato, R.D., Sarabia, R., Rivas, J.J., et al.: Normal computerized tomography scans in severe head injury. Prognostic and clinical management implications. 1986; 65: 784789.

18. Marshall, L.F.: Comment. Neurosurgery 2000; $46: 47$.

19. Marshall, L.F., Bowers, S.A., Klauber, M.R., et al.: A new classification of head injury based on computerized tomography. J Neurosurg 1991; 75: Suppl S14-S20.

20. Mayer, S.A., Brun, N.C., Begtrup, K., et al.: Recombinant activated factor VII for acute intracerebral hemorrhage. $\mathrm{N}$ Eng J Med 2005; 352: 777-785.
21. Narayan, R.K., Kishore, P.R., Becker, D.P. et al.: Intracranial pressure: to monitor or not to monitor. J Neurosurg 1982; 56: 650-659.

22. Oertel, M., Kelly, D.F., McArthur, D., et al.: Progressive hemorrhage after head trauma: predictors and consequences of evolving injury. J Neurosurg 2002; 96: 109-116.

23. Roberson, F.C., Kishore, P.R.S., Miller, J.D., Lipper, H.H., Becker, D.P.: The value of serial computerized tomography in the management of severe head injury. Surg Neurol 1979; 12: 161-167.

24. Sahuquillo, J., Biestro, A., Mena, P.M., et al.: Medidas de primer nivel en el tratamiento de la hipertensión intracraneal en el paciente con traumatismo craneoencefálico grave. Propuesta y justificación de un protocolo. Neurocirugía 2002; 13: 78-100.

25. Sawada, Y., Sadamitsu, D., Sakamoto, T., et al.: Lack of correlation between delayed traumatic intracerebral hematoma and disseminated intravascular coagulation. J Neurol Neurosurg Psychiatry 1984; 47: 1125-1127.

26. Servadei, F., Murray, G.D., Penny, K., et al.: The value of the "worst" computed tomographic scans in clinical studies of moderate and severe head injury. European Brain Injury Consortium. Neurosurgery 2000; 46: 70-77.

27. Servadei, F., Nanni, A., Nasi, M.T., et al.: Evolving brain lesions in the first 12 hours after head injury: Analysis of 37 comatose patients. Neurosurgery 1995; 37: 899-907.

28. Stein, S.C., Spettell, C., Young, G., Ross, S.E.: Delayed and progressive brain injury in closed-head trauma: radiological demonstration. Neurosurgery 1993; 32: 25-31.

29. Stein, S.C., Young, G.S., Talucci, R.C., et al.: Delayed brain injury after head trauma: significance of coagulopathy. Neurosurgery 1992; 30: 160-165.

30. Teasdale, G., Jennett, B.: Assessment of coma and impaired consciousness. A practical scale. Lancet 1984; 2: 8184.

31. Touho, H., Hirakawa, K., Hino, A., et al.: Relationship between abnormalities in coagulation and fibrinolysis and postoperative intracranial hemorrhage in head injury. Neurosurgery 1986; 19: 523-531.

32. van der Sande, Emeiss, J.J., Lindeman, J.: Intravascular coagulation: a common phenomenon in minor experimental head injury. J Neurosurg 1981; 54:21-25

Lobato, R.D.; Alen, J.F.; Perez-Nuñez, A.; Alday, R.; Gómez, P.A.; Pascual, B.; Lagares, A.; Miranda, P.; Arrese, I.; Kaen, A.: Utilidad de la TAC secuencial y la monitorización de la presión intracraneal para detectar nuevo efecto masa intracraneal en pacientes con traumatismo craneal grave y lesión inicial Tipo I-II. Neurocirugía 2005; 16: 217-234.

Correspondencia postal: RD Lobato. Servicio Neurocirugía. Hospital "12 de Octubre". Avda Cordoba/sn. 28041 Madrid. 